\title{
Imaging ellipsometry of graphene
}

\author{
Ulrich Wurstbauer, ${ }^{1, a), b)}$ Christian Röling, ${ }^{2}$ Ursula Wurstbauer, ${ }^{1, b)}$ Werner Wegscheider, ${ }^{1, c}$ ) \\ Matthias Vaupel, ${ }^{2, d}$ Peter $\mathrm{H}$. Thiesen, ${ }^{2}$ and Dieter Weiss ${ }^{1}$ \\ ${ }_{1}^{1}$ Institut für Experimentelle und Angewandte Physik, Universität Regensburg, 93040 Regensburg, Germany \\ ${ }^{2}$ Accurion GmbH, Stresemannstr. 30, 37079 Göttingen, Germany
}

(Received 18 August 2010; accepted 13 November 2010; published online 6 December 2010)

\begin{abstract}
Imaging ellipsometry studies of graphene on $\mathrm{SiO}_{2} / \mathrm{Si}$ and crystalline $\mathrm{GaAs}$ are presented. We demonstrate that imaging ellipsometry is a powerful tool to detect and characterize graphene on any flat substrate. Variable angle spectroscopic ellipsometry is used to explore the dispersion of the optical constants of graphene in the visible range with high lateral resolution. In this way, the influence of the substrate on graphene's optical properties can be investigated. (C) 2010 American Institute of Physics. [doi:10.1063/1.3524226]
\end{abstract}

Graphene is a two-dimensional crystalline solid consisting of only one atomic layer of hexagonally arranged carbon atoms. ${ }^{1}$ To the fascinating properties of this two dimensional lattice belong a record electron/hole mobility at room temperature and charge carriers behaving at low energies like massless Dirac fermions resulting in distinct transport properties such as half integer quantum Hall effect ${ }^{2,3}$ and Klein tunneling. ${ }^{4}$ Graphene has also a high potential for devices in various fields including, e.g., mechanically very "robust" transparent electrodes for touch screens, solar cells, photodetectors, nanoelectronics, and high frequency devices. ${ }^{5-7}$ For the latter, a combination with GaAs, currently used for high frequency applications, seems to be promising. However, the detection and characterization of graphene on GaAs-based materials has been reported to be very time-consuming ${ }^{8}$ or limited to special layered GaAs/AlGaAs heterostructures. ${ }^{9}$ It is well known that substrate and environment significantly influence graphene's electrical properties. ${ }^{10-12}$ Further, the influence of the substrate and environment on the optical properties of graphene has theoretically been predicted. ${ }^{13}$ As recently reported by Kravets et al., ${ }^{14}$ optical constants and the optical dispersion can be extracted from ellipsometric spectra. However, those experiments are limited to extremely large graphitic flakes due to the extended spot size of the light. Picometry allows investigations of the optical properties with a higher lateral resolution but is limited to certain wavelengths. ${ }^{15}$ Graphene oxide layers have already been characterized by imaging ellipsometry. ${ }^{16}$

In this letter, we demonstrate that imaging ellipsometric intensity (IEI) maps, imaging ellipsometry (IE), and imaging variable angle spectroscopic ellipsometry (IVASE) at visible light frequencies are powerful tools to detect and classify graphene flakes and to study their optical properties on a large variety of flat substrates. Due to the high lateral resolution of less than $1 \mu \mathrm{m}$ of our setup, the optical properties can be mapped over a graphitic flake. Here, we report on measurements of exfoliated graphene deposited either on

\footnotetext{
${ }^{a)}$ Electronic mail: ulrich.wurstbauer@physik.uni-regensburg.de.

${ }^{b}$ Present address: Department of Physics, Columbia University, USA.

${ }^{c}$ Present address: Solid State Physics Laboratory, ETH Zurich, 8093 Zurich, Switzerland.

${ }^{d)}$ Present address: Carl Zeiss MicroImaging GmbH, 37081 Göttingen, Germany.
}

$300 \mathrm{~nm}$ amorphous $\mathrm{SiO}_{2}$ on $\mathrm{Si}$ or GaAs-based substrates, grown by molecular beam epitaxy.

The graphene mono- and multilayer samples have been prepared by micromechanical exfoliation of natural graphite as introduced in Refs. 8, 17, and 18. The flakes were investigated by imaging ellipsometry under ambient conditions at room temperature with a nulling ellipsometer nanofilm_ep3se from Accurion $\mathrm{GmbH}^{19}$ using three different modes as described below. The optical properties are measured for wavelengths ranging from $\lambda=360 \mathrm{~nm}$ to $\lambda=1000 \mathrm{~nm}$ (bandwidth of \pm 6 to $\pm 20 \mathrm{~nm}$ ). The reflected light from the surface is focused with $20 \times$ or $50 \times$ objectives. The latter leads to a $68 \times 79 \mu \mathrm{m}^{2}$ field of view and enables a lateral resolution better than $1 \mu \mathrm{m}$, as demonstrated in Fig. 1(c). There, the two arrows mark a width of less than $800 \mathrm{~nm}$. In addition, size, shape, number of layers, morphology, and height of the graphene flakes have been determined by a combination of optical scanning electron microscopy (SEM) and atomic force microscopy (AFM).

In Fig. 1(a), the used imaging ellipsometry setup is schematically depicted. The angle of incidence (AOI) is the angle between the incident (reflected) light and the sample normal and can be varied. A polarizer polarizes the incoming light linearly which later gets elliptically prepared by a compensator in such a way that the reflected light is again linearly polarized. After passing an analyzer, the reflected light is collected by a charge coupled device (CCD) camera. The lens system enables the high lateral resolution. In the upper row of Fig. 1(a), the corresponding states of polarizations are sketched. In an appropriately chosen coordinate system, the ratio $\rho$ of the perpendicular $p$ and the orthogonal $s$ components of the reflection matrix can be described by $\rho=E_{\text {out }, p} / E_{\text {in }, p} / E_{\text {out }, s} / E_{\text {in }, s}=\tan (\psi) \cdot e^{i \Delta}$ with the ellipsometric angles $\Psi$ and $\Delta .{ }^{16,20,21} E_{\text {in(out), } p(s)}$ denotes the electric field of the incoming (outgoing) light parallel (orthogonal) to the plane of incident, as sketched in the top row of Fig. 1(a). In the IEI mode, the angle between polarizer and analyzer as well as the AOI is fixed and the intensity of the reflected light is mapped over the sample for a certain wavelength [see Fig. 1(c)]. For the IE mode, the intensity of the reflected light is minimized by a $90^{\circ}$ alignment of the analyzer (one zone nulling condition) and $\Psi$ and $\Delta$ values are plotted. In IVASE mode, $\Psi$ and $\Delta$ values are determined in dependence of the AOI and wavelength of the incident light. This information 


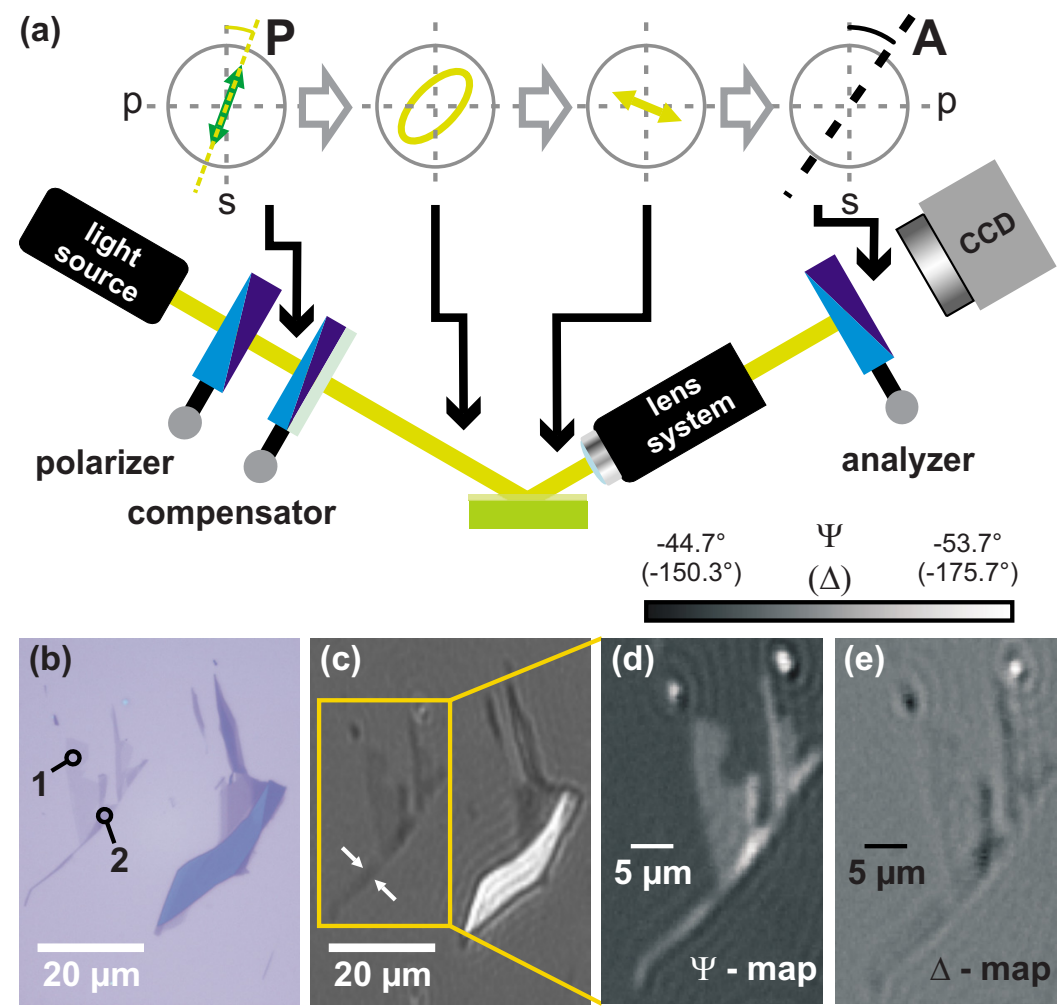

FIG. 1. (Color online) (a) Schematic imaging ellipsometry setup. The lens system mounted between the sample and analyzer allows imaging with submicron lateral resolution. (b) Optical image and (c) imaging ellipsometric intensity image of a sample on $\mathrm{SiO}_{2} / \mathrm{Si}$ showing regions with graphene monolayer covering up to thin graphite. Numbers in (b) correspond to the layer number. (d) Ellipsometric $\Psi$ map and (e) the corresponding $\Delta$ map of the boxed region display graphene mono- and bilayer areas with higher resolution.

enables to determine the dielectric function of graphene.

An optical micrograph of graphene flakes with different layer numbers ranging from monolayer up to few-layer graphene and thin graphite is shown in Fig. 1(b). Employing the well established contrast method, ${ }^{22,23}$ the part of the left flake is identified as mono- and bilayer graphenes. An IEI gray color plot of these graphitic sheets on $\mathrm{SiO}_{2} / \mathrm{Si}$ taken with a $20 \times$ objective with $\mathrm{AOI}=42^{\circ}$, a polarizer angle of $63.80^{\circ}$, and an analyzer angle of $48.21^{\circ}$ (compensator at $45^{\circ}$ ) using $552 \mathrm{~nm}$ light is displayed in Fig. 1(c). The gray scale reflects the real intensities captured by the CCD camera. Brighter regions, therefore, correspond to polarization changes of the reflected light that match the analyzer angle better than darker regions. Comparing Figs. 1(b) and 1(c) illustrates that the contrast in the IEI plot is appropriate to differentiate between graphene mono-, bi-, and few-layer. The sheets are better visible in the IEI image and both shape and layer number are clearly distinguishable by IEI. The height of the monolayer region was determined by AFM to be about $0.7 \mathrm{~nm}$. The difference to the commonly accepted graphene height deduced from graphite's interlayer distance may be explained by a layer of water below the sheet as recently demonstrated. ${ }^{24}$ Figures $1(\mathrm{~d})$ and $1(\mathrm{e})$ display IE $\Psi$ and $\Delta$ maps $\left(\mathrm{AOI}=42^{\circ}\right)$ of the framed region of Fig. $1(\mathrm{c})$. The shape of the flake is unambiguously visible in the $\Psi$ as well as in the $\Delta$ map; however, the $\Psi$ map gives a clearer signal for the monolayer region, whereas the $\Delta$ map displays a stronger one for the bilayer region.

On substrates other than $\mathrm{SiO}_{2} / \mathrm{Si}$, graphene can hardly be detected with an optical microscope. ${ }^{25}$ As a demonstration of IEI's capability to determine the shape and number of graphene layers on any flat substrate, we have investigated graphene on crystalline GaAs. In Fig. 2, a graphene flake (marked with circles), deposited on the surface of GaAs, is imaged by (a) SEM, and in (b) and (c) by IEI maps with different fixed angles between polarizer and analyzer at a wavelength of $532 \mathrm{~nm}$. In the SEM image, the flake and the surrounding "L-shaped" resist/glue residues give a strong contrast and only the straight edges of the flake enable to distinguish residues and graphene. The image shown in Fig. 2(b) was taken with an angle of $12.936^{\circ}$ between polarizer and analyzer resulting in a large signal of the approximately $10 \mu \mathrm{m}$ long flake while the resist/glue residues are faint. Interestingly, a minor change of the angle to $19.095^{\circ}$ in Fig. 2(c) gives the opposite result: the contrast of the L-shaped residues is enhanced while the flake image fades away.

Under the assumption that the dielectric constants of graphene are constant and that the thickness of the layer is the only free fit parameter, the $\Psi$ map around the graphene sheet shown in Fig. 2(b) can be converted into a thickness map. The line profile along the red line is shown in the inset of Fig. 3(a). The height of about $2.1 \mathrm{~nm}$ and the profile of this few-layer graphene flake are in good agreement with the corresponding AFM profile.

Besides visualizing the flakes on various substrates, IVASE allows to determine the optical properties of thin films. To extract the optical constants of graphene monolay-

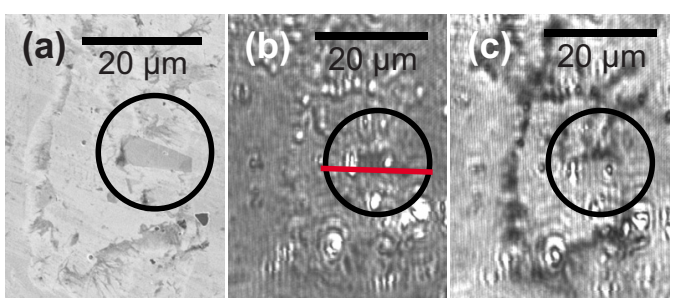

FIG. 2. (Color online) (a) SEM image of few-layer graphene on a GaAs substrate. The flake is centered in the circle and in the surroundings are resist/tape residues. (b) and (c) are IEI plots of the same region. In (b) the contrast is optimized for the graphene layer such that the adhesive tape residues vanish, while in (c) the contrast of the immediate vicinity is enhanced. 


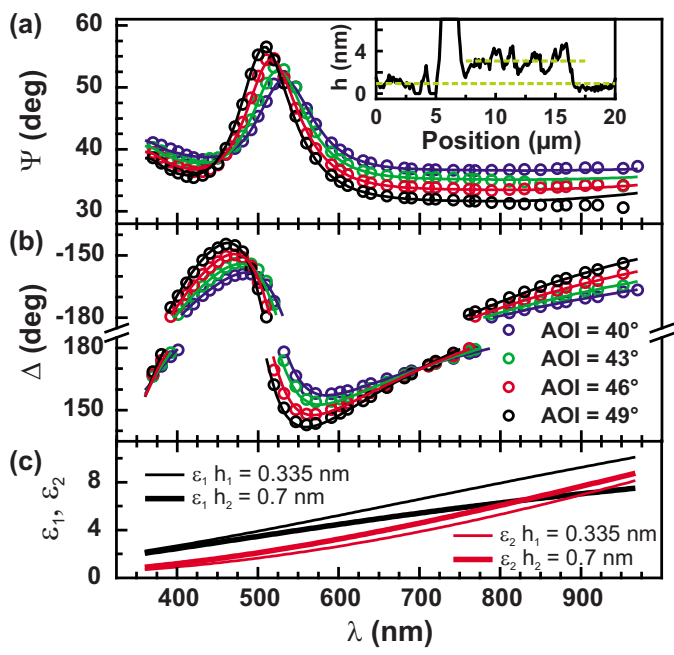

FIG. 3. (Color online) (a) Wavelength dependent $\Psi$-angle of a graphene monolayer for different AOIs. Inset: height profile of the flake on GaAs shown in Fig. 2. (b) Wavelength dependency of the $\Delta$-angle for the same AOI as in (a). (c) Dielectric coefficients $\epsilon_{1}$ and $\epsilon_{2}$ of graphene for both theoretical layer height $h_{1}=0.335 \mathrm{~nm}$ (thin lines) and AFM measured height $h_{2}=0.7 \mathrm{~nm}$ (thick lines).

ers, several regions of interest (ROIs) are carefully chosen from the IEI of the graphene monolayer displayed in Fig. 1(c). $\Psi$ and $\Delta$ values were measured for wavelengths between $\lambda=370 \mathrm{~nm}$ and $\lambda=952 \mathrm{~nm}$ at AOIs of $40^{\circ}, 43^{\circ}$, $46^{\circ}$, and $49^{\circ}$. The resulting values are averaged over all pixels of the CCD chip representing each ROI and collected in Figs. 3(a) and 3(b). We made sure that the ROI was fully centered on a graphene flake as uncovered substrate contributes to the IVASE results. Consequently, the investigated regions are centered on the graphene and the AOI was small enough $\left(\sim 45^{\circ}\right)$ to ensure that the measured spot is only placed on the graphene flake. As evident from Figs. 3(a) and 3(b), both $\Psi$ and $\Delta$ depend on the used wavelength. The $\Psi$ values are less affected above $\lambda=600 \mathrm{~nm}$, while a maximum develops at about $500 \mathrm{~nm}$. The $\Delta$ curves pass a local minimum below $\lambda=575 \mathrm{~nm}$ and a maximum around $\lambda=475 \mathrm{~nm}$. The extremal values change slightly with the AOI. The experimental obtained values are in good agreement with the findings in Ref. 14. The optical parameters have been modeled with the NANOFILM_EP4MODEL software based on the Fresnel coefficients for multilayered films and on the Drude model, provided by Accurion GmbH. ${ }^{16,21,26}$ The data have been fitted using two different values for the graphene monolayer thickness: the interlayer distance in graphite $h_{1}=0.335 \mathrm{~nm}$ and the AFM measured value for the investigated flake of $h_{2}=0.7 \mathrm{~nm}$. The relative root mean square error of the fits is $27.4 \%$ and $1.4 \%$ for $h_{1}$ and $h_{2}$, respectively. The best fit results using the measured physical instead of the theoretical value of the graphene monolayer thickness. For completeness, the determined dielectric coefficients $\epsilon_{1}$ and $\epsilon_{2}$ are shown in Fig. 3(c) for both $h_{1}$ (thin lines) and $h_{2}$ (thick lines). Both coefficients increase with increasing wavelength.

Spectroscopy of $\Psi$ and $\Delta$ was also done on the few-layer graphene flake on GaAs, shown in Fig. 2. The $\Delta$ values show a similar dependence on wavelength as found for graphene monolayers on $\mathrm{SiO}_{2}$, whereas the maximum of the $\Psi$ map seems to be shifted to wavelengths below $400 \mathrm{~nm}$. The origin of these differences is unknown yet. They could either be caused by the different substrate materials or by increasing the layer number from monolayer (on $\mathrm{SiO}_{2}$ ) to few-layer graphene (on GaAs). Modeling our data results in a similar dispersion, as reported in Ref. 15, and shows the same trend as reported in Ref. 14. The origin of the quantitative difference in the extracted optical dispersion is not yet clear. A height independent way would be four-zone nulling spectroscopic ellipsometry, which is, however, out of the scope of this letter.

In conclusion, it has been shown that shape and layer number of exfoliated graphene sheets can be determined on amorphous insulating $\mathrm{SiO}_{2}$ and crystalline semiconducting GaAs substrates by IE. From IVASE, the optical properties can be extracted. This method enables to proof the prediction that the optical properties of graphene are dependent from the substrate. Furthermore, changes of the optical properties by including imperfections, e.g., by patterning of antidot lattices, ${ }^{27}$ or by the edges could be explored with IE.

\section{Work supported by DFG via GK 638 and GK 1570.}

${ }^{1}$ A. H. Castro Neto, F. Guinea, N. M. R. Peres, K. S. Novoselov, and A. K. Geim, Rev. Mod. Phys. 81, 109 (2009).

${ }^{2}$ K. S. Novoselov, A. K. Geim, S. V. Morozov, D. Jiang, M. I. Katsnelson, I. V. Grigorieva, S. V. Dubonos, and A. A. Firsov, Nature (London) 438, 197 (2005).

${ }^{3}$ Y. Zhang, Y. Tan, H. Stormer, and P. Kim, Nature (London) 438, 201 (2005).

${ }^{4}$ A. F. Young and P. Kim, Nat. Phys. 5, 222 (2009).

${ }^{5}$ S. Bae, H. Kim, Y. Lee, X. Xu, J.-S. Park, Y. Zheng, J. Balakrishnan, T. Lei, H. Ri Kim, Y. I. Song, Y.-J. Kim, K. S. Kim, B. Ozyilmaz, J.-H. Ahn, B. H. Hong, and S. Iijima, Nat. Nanotechnol. 5, 574 (2010).

${ }^{6}$ F. Bonaccorso, Z. Sun, T. Hasan, and A. C. Ferrari, Nat. Photonics 4, 611 (2010).

${ }^{7}$ Y. Lin, K. A. Jenkins, A. Valdes-Garcia, J. P. Small, D. B. Farmer, and P. Avouris, Nano Lett. 9, 422 (2009).

${ }^{8}$ U. Stöberl, U. Wurstbauer, W. Wegscheider, D. Weiss, and J. Eroms, Appl. Phys. Lett. 93, 051906 (2008).

${ }^{9}$ M. Friedemann, K. Pierz, R. Stosch, and F. J. Ahlers, Appl. Phys. Lett. 95, 102103 (2009)

${ }^{10}$ C. Dean, A. F. Young, I. Meric, C. Lee, L. Wang, S. Sorgenfrei, K. Watanabe, T. Taniguchi, P. Kim, K. Shepard, and J. Hone, Nat. Nanotechnol. $\mathbf{5}, 722(2010)$.

${ }^{11}$ K. I. Bolotin, K. J. Sikes, Z. Jiang, M. Klima, G. Fudenberg, J. Hone, P. Kim, and H. L. Stormer, Solid State Commun. 146, 351 (2008).

${ }^{12}$ X. Hong, A. Posadas, K. Zou, C. H. Ahn, and J. Zhu, Phys. Rev. Lett. 102, 136808 (2009).

${ }^{13}$ V. Meera and G. S. Setlur, J. Appl. Phys. 107, 033525 (2010).

${ }^{14}$ V. G. Kravets, A. N. Grigorenko, R. R. Nair, P. Blake, S. Anissimova, K. S. Novoselov, and A. K. Geim, Phys. Rev. B 81, 155413 (2010).

${ }^{15}$ X. Wang, Y. P. Chen, and D. D. Nolte, Opt. Express 16, 22105 (2008).

${ }^{16}$ I. Jung, M. Vaupel, M. Pelton, R. Piner, D. A. Dikin, S. Stankovich, J. An, and R. S. Ruoff, J. Phys. Chem. C 112, 8499 (2008).

${ }^{17}$ K. S. Novoselov, D. Jiang, F. Schedin, T. Booth, V. Khothevicha, S. Morozov, and A. Geim, Proc. Natl. Acad. Sci. U.S.A. 102, 10451 (2005).

${ }^{18}$ K. S. Novoselov, A. K. Geim, S. V. Morozov, D. Jiang, Y. Zhang, S. V Dubonos, I. V. Grigorieva, and A. A. Firsov, Science 306, 666 (2004).

${ }^{19}$ Accurion GmbH, Stresemannstr. 30, 37079 Göttingen, Germany, http:// www.nanofilm.de.

${ }^{20}$ O. S. O. America, Handbook of Optics: Devices, Measurements, and Properties, 2nd ed. (McGraw-Hill Professional, New York, 1994), Vol. 2.

${ }^{21}$ Handbook of Ellipsometry, edited by H. G. Tompkins and E. A. Irene (William Andrew/Noyes, Norwich, 2005).

${ }^{22}$ D. S. L. Abergel, A. Russell, and V. I. Fal'ko, Appl. Phys. Lett. 91, 063125 (2007).

${ }^{23}$ P. Blake, E. W. Hill, A. H. C. Neto, K. S. Novoselov, D. Jiang, R. Yang, T. J. Booth, and A. K. Geim, Appl. Phys. Lett. 91, 063124 (2007).

${ }^{24}$ K. Xu, P. Cao, and J. R. Heath, Science 329, 1188 (2010).

${ }^{25}$ X. Wang, M. Zhao, and D. D. Nolte, Appl. Phys. Lett. 95, 081102 (2009).

${ }^{26}$ H. G. Tompkins and W. A. Mcgahan, Spectroscopic Ellipsometry and Reflectometry: A User's Guide (Wiley-Interscience, New York, 1999).

${ }^{27}$ J. Eroms and D. Weiss, New J. Phys. 11, 095021 (2009). 